\title{
Chemical and petrographic characterization of amorphous silicate material in cometary GEMS
}

\author{
BIRGIT SCHULZ ${ }^{1}$, CHRISTIAN VOLLMER ${ }^{2}$, JAN \\ LEITNER $^{3}$ AND LINDSAY P. KELLER ${ }^{4}$ \\ ${ }^{1}$ Universität Münster \\ ${ }^{2}$ Institut für Mineralogie, Universität Münster \\ ${ }^{3}$ Max Planck Institute for Chemistry \\ ${ }^{4}$ NASA Johnson Space Center \\ Presenting Author: b_schu64@uni-muenster.de
}

Amorphous silicate material (ASM) is found in the matrix of GEMS (glass with embedded metal and sulfides) in chondritic porous interplanetary dust particles (CP-IDPs) [1] and in the matrices of primitive chondritic meteorites [2]. It either formed by irradiation of crystalline precursors in the interstellar medium [3] or by non-equilibrium condensation in the solar nebula [1]. However, the origins of ASM in IDPs and chondrites and their possible relationship are still not well constrained. Here we performed a petrographic and chemical characterization of ASM in five CP-IDPs by Transmission Electron Microscopy (TEM) techniques. We also analyzed carbon and nitrogen isotopic compositions of organic matter by NanoSIMS to constrain the pristine character of analyzed IDPs. ASM in IDPs is depleted in all major element/Si ratios with respect to the solar value. Compared to bulk GEMS analyses [4], the ASM contains less $\mathrm{Fe}$, because nanophase inclusions of Fe-Ni-metal and $\mathrm{FeS}$ are mostly excluded by our approach. In contrast, ASM in meteorites has higher-than-solar $\mathrm{Fe} / \mathrm{Si}$ ratios, maybe due to aqueous alteration on the meteorite parent bodies [2,5]. Chemical composition of GEMS ASM indicates a non-equilibrium condensation origin. Silicone oil contamination of particles during collection is excluded, because GEMS collected in dry polyurethane show similar compositions [6]. Magnetite rims around GEMS in our IDP samples indicate heating of some particles during atmospheric entry. However, preserved ${ }^{15} \mathrm{~N}$ and ${ }^{13} \mathrm{C}$ isotopic anomalies in organic matter around GEMS and in IDP matrix link the IDPs to a cold environment and overall validate their primitiveness [7].

Acknowledgements: We thank NASA's Astromaterials Acquisition and Curation Office for providing IDP samples, and the DFG for funding this project (VO1816/5-1).

References: [1] Keller, L.P. \& Messenger, S. (2011), Geochim. Cosmochim. Acta 75(18), 5336. [2] Hopp, T. \& Vollmer, C. (2017), Meteorit. Planet. Sci. 53(2): 153. [3] Bradley, J.P. \& Dai, Z.R. (2004), ApJ 617: 650. [4] Bradley, J.P. (1994), Science 265(5174), 925. [5] Vollmer, C. et al. (2020), Meteorit. Planet. Sci. 55(7), 1491, [6] Messenger et al. (2015), Meteorit. Planet. Sci. 50(8), 1468. [7] Floss, C. et al. (2006), Geochim. Cosmochim. Acta 70(9), 2371. 